\title{
PERSPECTIVAS PROFESIONALES \\ DE LOS ALUMNOS DE BACHILLERATO \\ EN EL ESTADO DE HIDALGO.
}

Gregorio Sánchez Muñoz*

\section{RESUMEN}

En este trabajo se pretende conocer las preferencias profesionales del alumnado que está cursando el $5^{\circ}$ y $6^{\circ}$ semestres de bachillerato, se llevó a cabo una encuesta en varios municipios de la entidad en escuelas públicas y privadas. Se obtuvieron resultados acerca de las carreras más demandadas, así como de las instituciones con mayor preferencia.

\section{ABSTRACT}

It is intended to know from this project the professional preferences of the alumni between fifth and sixth semester in high school, a survey was applied in different municipalities of the state in public and private schools. Results obtained pointed the most demanded careers, as well as the most preferred institutions.

Estudio realizado durante el ciclo escolar 2007-2008

Coordinador de Investigación de la Universidad La Salle Pachuca. investigacion@lasallep.edu.mx

Recibido 25-XI-2008 * Aceptado 9-I-2009 * Corregido 3-II-2009

Palabras clave: preferencias profesionales, bachillerato, motivaciones escolares, licenciaturas, escuelas públicas y privadas.

Key words: professional preferences, high school, schooling motivation, bachelor degrees, private and public schools. 


\section{INTRODUCCIÓN}

Las interrogantes del estudiante, una vez que ha rebasado los niveles de educación que anteceden a la educación superior son: ¿qué carrera elegir? ¿Se ofrece la carrera en la entidad? ¿Qué instituciones la ofertan?

¿Qué factores debo considerar para elegir tal o cual institución? También están los cuestionamientos que se hacen las instituciones de educación superior (IES) en relación con las expectativas del estudiantado y llenar sus aulas en el siguiente ciclo escolar sobre todo, si de instituciones privadas se trata.

En busca de respuestas, la Universidad La Salle Pachuca, ha realizado algunos estudios, con el objetivo de conocer las preferencias profesionales del alumnado que cursa el nivel medio superior de la entidad. En el presente trabajo, se dan a conocer resultados de estudios realizados en diferentes ciclos escolares, y, con mayor detalle una indagación que se hizo al inicio del presente ciclo escolar 2007-2008. Se partió de da- tos oficiales. Según el Sistema de Educación Pública de Hidalgo (SEPH), Estadística Básica Educativa (EBE), la matrícula registrada en el ciclo escolar 2006-2007; la población estudiantil en la entidad en el nivel bachillerato ascendía a 95,083 alumnos. De esta cifra, 26 . $\%,\left(24,858\right.$ corresponden a los semestres $5^{\circ}$ y $\left.6^{\circ}\right)$.

El objetivo del estudio fue identificar las preferencias profesionales de los estudiantes, por lo que se aplicó un cuestionario en algunas escuelas de este nivel. La muestra fue selectiva y voluntaria lo que significó una limitante. La muestra abarcó a 2755 estudiantes de $5^{\circ}$ y $6^{\circ}$ semestres, sobre todo de $5^{\circ}$. Dicha cifra representa . \% de la población estudiantil. El trabajo de campo se realizó en septiembre y octubre de 2007. En escuelas públicas hubo 559 matriculados (56.6\%) de la muestra y en privadas $96(43.4 \%)$ de la muestra. Algunas instituciones no aparecen por la tardanza en su respuesta acerca del otorgamiento del permiso correspondiente, otras por lo general negaron el acceso. 


\section{Escenario Educativo}

La situación educativa en la entidad, particularmente en el nivel superior ha tenido una evolución vertiginosa, tanto en la proliferación de IES, como el aumento de programas académicos. En este sentido y con base en la información de la Dirección de Educación Superior del Instituto Hidalguense de Educación Media Superior y Superior (IHEMSYS) ${ }^{2}$; muestran datos de la última década del siglo pasado. Subrayan que de 99 a 2000 el número de instituciones o campus que integran el Sistema Superior en Hidalgo, aumentó de seis a 3, sin incluir a las es- cuelas normales. En los últimos siete años (ciclos escolares 993- 994 a 999-2000) la población de nuevo ingreso a educación superior en el Estado, pasó de ,700 alumnos a 6,900, lo que representa un crecimiento promedio anual del $22.2 \%$.

En el periodo comprendido de 99 a 999 se incrementó el número de programas de educación superior en $82 \%$, al pasar de 40 a 73 , sobresaliendo el área de Ciencias Sociales y Administrativas 3 con 5 nuevos programas, la de Educación con siete e Ingeniería y Tecnología con seis.

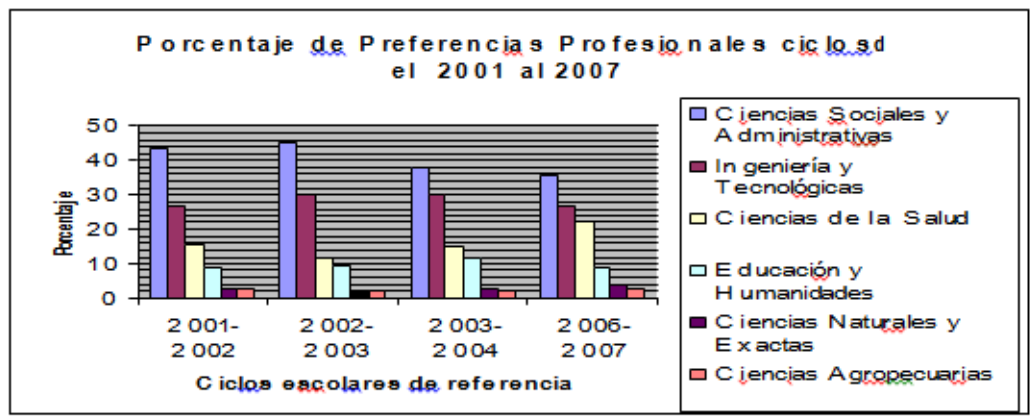


2. Sujetos objetos de estudio:

Se aplicaron los cuestionarios al alumnado que asistió el día de la encuesta: $93.7 \%$ del quinto semestre y el $6.3 \%$ del sexto semestre. Resultados: $40.7 \%$ son hombres y $59.3 \%$ mujeres. Inferencia de cada 0 , seis son del sexo femenino. El $60 \%$ del alumnado promedia 7 años. El $20 \%$ son menores de 7 años, $9 \%$ tiene entre 8 y 20 años. Sólo el $\%$ son mayores de 2 años.

En cuanto al nivel de ingreso de las familias de los encuestados. Dos de cada diez familias ganan hasta $\$ 4000.00$ mensuales; en contraste nueve de cada cien gana $\$ 6,000.00$ o más. Otro dato relevante es que el $4 \%$ desconoce a cuánto asciende el ingreso de su familia.

3. Información obtenida de la encuesta

3. Área propedéutica y disciplinar preferida: Sin considerar los que cursan el bachillerato general, tanto de escuelas públicas, como privadas, sigue el dominio de las ciencias sociales y administrativas con el $28.3 \%$, en contraste con las llamadas ciencias duras que sólo registran el $5.4 \%$. Evidentemente, los efectos se observan en las licenciaturas, sobre todo en las carreras tradicionales de ciencias sociales 4 las cuales siguen saturadas, no así las ciencias exactas e ingenierías. En este sentido, sigue pendiente la indagación, ¿por qué se desdeña estudiar disciplinas vinculadas con la física, matemáticas y por ende las ingenie- rías? Se infiere que es herencia desde la educación básica, si se revisa lo que ocurre con el área de aritmética y geometría en primaria; lo que sucede con matemáticas y física en el nivel secundaria. 5 La respuesta parece obvia. Aunque queda pendiente hacer un análisis más profundo de esta información. 
3.2. Perspectivas del egresado y absorción de IES: Resulta gratificante observar que el $98.7 \%$ desea continuar estudios de licenciatura. Contrastando esta aseveración con los datos de ANUIES, a nivel nacional: de cada 0 matriculados en bachillerato sólo siete se registran en los niveles superiores. En el nivel estatal con información del Sistema de Educación del Estado de Hidalgo (SEPH), se hizo un análisis de los egresados únicamente. Durante los últimos cinco ciclos escolares: $2001-2002$ al

2006-2007. Nivel bachillerato, población de egreso, crecimiento anual (7. \%) en promedio. En cuanto a población egresada anualmente, durante ese mismo periodo, oscila en los 7700 jóvenes.

El sector público, en este nivel, atiende ocho de cada diez. De esta cifra, el gobierno estatal se encarga del $30 \%$, con crecimiento anual de absorción importante. También se observa que uno de cada cinco que se matriculan en el nivel referido, lo hacen en instituciones privadas. Anualmente en promedio 7500 jóvenes y señoritas se inscriben en otras áreas educativas, educación normal, capacitaciones para el trabajo o se incorporan al campo laboral. La cifra resulta importante ya que en un lapso de cinco años, rebasa los 35000 estudiantes. Retomando los egresados. De ese $70 \%$, en cuanto al porcentaje promedio de absorción que han tenido las IES durante los últimos cinco ciclos escolares, se puede dar a conocer lo siguiente: a) En conjunto, los Organismos Descentralizados del Gobierno del Estado 6 absorben el $29.9 \%$. b) Por su parte, la Universidad Autónoma del Estado de Hidalgo y sus campi en la entidad, incorporan el $28.7 \%$. c) Las instituciones privadas atienden un promedio de $30 \%$, con un crecimiento promedio anual del $.9 \%$. 
En este nivel educativo, se puede apreciar que el $70 \%$ es atendido por alguna instancia del sector público, es decir, siete de cada 0 se matriculan en dicho sector. El $30 \%$ restante lo absorben las IES privadas, pero con un crecimiento promedio anual del $2 \%$, muy por encima de lo que crecen las IES públicas.

3.3. Motivos para estudiar: Los motivos que más sobresalen para estudiar una carrera y en esto coinciden los matriculados en ambos sectores-instituciones públicas y privadas- están: en primer término: a) poder trabajar en lo que les gusta $6.8 \%$; b) considerar que se gana más dinero el $22.0 \%$; c) es más fácil que otras carreras, y por imagen que refleja el profesional, ambas con el $5 \%$ cada una. Prácticamente nula como motivación importante la existencia o no del campo de trabajo $(0.9 \%)$.

No obstante el crecimiento en número de IES, así como de la oferta educativa a nivel superior en la entidad (nuevas carreras) y según datos del SEPH -el número de IES en el ciclo 2002-2003, sumaban 30, de éstas, 8 eran particulares. Para el ciclo 20062007, la situación cambió: 4 IES,

27 particulares. Dos escuelas públicas nuevas y nueve privadas más. Se abrieron casi dos escuelas privadas por año en promedio- el $3.7 \%$ del alumnado desea buscar otras carreras que no se ofrecen aquí, sobre todo preferían la Universidad Nacional Autónoma de México (UNAM).

3.4. Carreras con mayor preferencia: Las carreras elegidas son similares a las que eligieron en su momento, sus colegas de generaciones anteriores. En este estudio, se hace un análisis entre escuelas del régimen público y privado. La carrera de médico cirujano es elegida con el mismo porcentaje en ambos regímenes y continúa siendo el de mayor preferencia; seguida por la de abogado, aunque la preferencia está de dos a uno de los matriculados en escuelas particulares y las públicas, respectivamente.

Otra licenciatura preferida ubicada en el tercer sitio, es administración, ésta la prefieren un poco menos los estudiantes de las escuelas privadas, que las públicas.

En términos generales, las licenciaturas con mayor preferencia y que eligen más para cursarla los alumnos (as) de las escuelas privadas, en relación con las escuelas públicas son: derecho, arquitectura, gastronomía, ciencias de la educación, diseño gráfico, ciencias de la comunicación, comercio internacional, pedagogía y mercadotecnia. Por su parte, los matriculados en escuelas públicas prefieren más las licenciaturas de administración, psicología, turismo, ingeniero mecánico, contaduría, ingeniería civil, ingeniería cibernética y sistemas computacionales, ingeniería industrial, y enfermería. Queda pendiente saber ¿cuáles son las razones y motivos de estas inclinaciones hacia carreras considera- das tradicionales? 
3.5. Preferencias por las escuelas y los motivos de dicha preferencia: La UAEH es preferida por el $37.4 \%$, le sigue el Instituto Tecnológico de Pachuca con un modesto $9.4 \%$. Ambas son preferidas por el $46.8 \%$. Con respecto a las instituciones privadas: el $7.8 \%$ tiene preferencia por el ITESM, seguido por La Salle Pachuca con el $7.2 \%$, el CENHIES con el $4.6 \%$ y la Universidad del Fútbol con el $2.0 \%$. En conjunto las cuatros IES privadas son preferidas por el $2.6 \%$ de los encuestados. Haciendo la sumatoria de públicas y privadas, las seis IES, tienen la preferencia del

$68.4 \%$ de la población encuestada; sin soslayar que de este porcentaje, más de la mitad corresponde a la UAEH.

En cuanto a la selección de la IES se pudo saber que poco más del $50 \%$ del alumnado elige porque supone o tiene conocimiento que dicha institución tiene un buen nivel académico y un prestigio ganado, también porque tiene la licenciatura que desea cursar. La cercanía de la escuela sólo lo considera el $5 \%$ de los encuestados y la tradición familiar escasamente el 3\%.

3.6. Difusión de la oferta educativa: Uno de los rubros que deberá trabajarse en la entidad, es la difusión de la oferta que existe en las IES. La mayoría de los encuestados desconoce la existencia de IES y programas educativos que se ofrecen en la entidad.

\section{CONCLUSIONES}

Con base en la experiencia y los resultados obtenidos en el estudio, se puede comentar que hace falta ampliar la difusión de la oferta educativa de nivel superior existente en la entidad. El alumnado debe conocer la existencia de las IES, su ubicación, características de infraestructura física: instalaciones, reconocimiento oficial, plantilla docente, modalidad educativa, programas académicos que ofrece y costos, entre otros aspectos. De esta forma, lo alumnos podrán elegir con mayor objetividad y cumplir sus expectativas. Deben recibir orientación vocacional adecuada y profunda, saber qué se requiere para estudiar dicha carrera y lo que implica trabajar en ella, de esa forma tendrá mayor certeza de lo que busca y no cambiar de carrera porque realizó una mala elección. Se considera que las instancias educativas gubernamentales podrían apoyar un programa de difusión de ese tipo. Podría hacerse un estudio similar a éste más completo, donde se abarquen todas las instituciones tanto públicas como privadas, también apoyadas por autoridades en la materia.

\section{NOTAS}

IHEMSYS. Opciones profesionales de Educación Superior, 2001, pp. 4 -46. 
2 Para la clasificación de las áreas de conocimiento se tomó en consideración lo que establece la Asociación Nacional de Universidades e Instituciones de Educación Superior.

3 Derecho, Administración, Contaduría, Psicología entre otras.

4 En nota del 24 de agosto de 2007, de Nurit Martínez del periódico El Universal, con el título "Bajo nivel educativo en $80 \%$ de niños: SEP". Escribe: De acuerdo con los resultados de la Evaluación Nacional de Logros Académicos en Centros Escolares (ENLACE), el nivel de conocimiento y aprendizaje de los niños de primaria y secundaria en español y matemáticas va de "insuficiente a elemental" en cerca de 8 millones de alumnos de los poco más de 0 millones que evaluó la Secretaría de Educación Pública (SEP). La prueba indica que 77.7\% (6 millones 634 mil estudiantes) de primaria obtuvieron un desempeño de "in- suficiente a elemental" en matemáticas, mientras en español la proporción de quienes salen en ese "grupo" de calificación es $75.4 \%$ (6.4 millones alumnos). En el caso de los jóvenes evaluados en secundaria, $94.4 \%$ de ellos (un millón

520 mil alumnos) obtuvieron calificación "insuficiente y elemental" en matemáticas; mientras .3 millones tuvo el mismo desempeño en español. Según el mismo reporte de ENLACE sólo 8 mil jóvenes $(0.5 \%)$ de alumnos tuvo un examen "excelente" en matemáticas; y apenas \%, calificación "perfecta" en español. Finaliza su texto con lo siguiente: Los desempeños más bajos se presentaron en Chiapas, Guerrero, Hidalgo, Nayarit, Oaxaca, Puebla, San Luis Potosí, Tabasco y Zacatecas.

$5 \quad$ Universidades Tecnológicas, Universidades Politécnicas, Institutos Tecnológicos Superiores.

\section{FUENTES DE CONSULTA}

- ANUIES. (2003). Anuarios Estadísticos de Licenciatura. México: Asocia- ción Nacional de Universidades e Instituciones de Educación Superior.

- IHEMSYS. (200 ). Opciones profesionales de Educación Superior. Pa- chuca, Hidalgo: Secretaría Educación Pública Hidalgo.

- La Salle Pachuca (200 , 2002, 2003). Preferencias profesionales. México: Boletín de la Coordinación de Investigación de La Salle Pachuca.

- Sistema Estatal de Educación: http://intranet.e-hidalgo.gob.mx/seph/. (noviembre y diciembre 2007). Estadística básica. De 200 -2002 al 2006-2007). 